\title{
The Influence of a Plane Boundary on the Thermophoretic Force in the Knudsen Regime
}

\author{
M. M. R. WILLIAMS \\ Nuclear Engineering Department, The University of Michigan, Ann Arbor, Michigan 48109
}

Received June 1, 1986; accepted July 18, 1986

\begin{abstract}
The effect of a plane diffusely reflecting wall on the thermophoretic force acting on a particle in the Knudsen regime is investigated. A general expression for the force on a particle in a nonuniform gas is given and then the velocity distribution function for the temperature jump problem is inserted. This results in an expression for the thermophoretic force that is a function of the distance of the particle from the wall. At large distances from the wall, the classic results of Waldmann and Schmitt are obtained, but near the wall there is a small reduction of about $1 \%$ in this value. The wall correction is independent of the gas-particle interaction, at least for specular, diffuse, Lambert, and backward scattering. The temperature jump problem has been solved using a collision cross section that is independent of velocity. This model, with diffuse reflection at the wall, predicts that the effect of the wall on the thermophoretic force becomes negligible at distances greater than a few mean free paths from the surface. () 1987 Academic Press, Inc.
\end{abstract}

\section{INTRODUCTION}

A particle suspended in a gas containing a temperature gradient will experience a force which normally drives the particle in the direction of decreasing temperature. This phenomenon is known as thermophoresis and has been the subject of considerable study for many years $(1,2)$. The explanation of the effect is based upon kinetic theory arguments and depends upon the fact that the particle receives a greater number of molecular impacts on its hotter side than on its colder one, thus leading to a net rate of change of momentum in the direction opposite to the temperature gradient. Additional mechanisms have been postulated to explain thermophoresis and a variety of second-order effects have been included $(3,4)$, but we shall not dwell on those matters here. It is important to note, however, that the magnitude of the effect depends upon the ratio of the thermal conductivities of the particle and of surrounding fluid as well as on the Knudsen number. For large Knudsen numbers, Waldmann and Schmitt (5) have given an extensive discussion of ways to obtain both the thermophoretic force and the corresponding thermophoretic velocity. Williams (6) has shown how the nature of the gas-surface interaction influences the thermophoretic effect by introducing a global scattering kernel for the aerosol particle. In the limit of small Knudsen numbers, Epstein (7) developed a method for obtaining thermophoretic forces using the continuum equations of fluid flow in the viscous flow limit, together with the classical equation of thermal conduction. His technique has been improved and extended to large Knudsen numbers by the addition of velocity slip and temperature jump boundary conditions (8).

In all of the work reported to date, the thermophoretic forces have been calculated on isolated particles in an infinite gas. The effect of boundaries has been completely neglected. This is in some ways a curious omission since it is the deposition onto boundaries that constitutes the major practical consequence of thermophoresis. Nevertheless, the interaction between the particle, the boundary, and the fluid has been neglected. Recently, however, 
the author (9) has examined, in the limit of small Knudsen numbers, how a plane boundary affects the thermophoretic force on a spherical particle. Significant effects are noted particularly at distances within two or three particle diameters. It is the purpose of the present paper to examine this same problem in the limit of large Knudsen numbers. Now the main physical difference between this and the hydrodynamic case is that, due to the smallness of the particle compared with a mean free path, the presence of the particle does not disturb the gas atom velocity distribution function. Thus, knowing the solution of the Boltzmann equation for the situation under consideration, it is easy to obtain the net force on the particle. In the case of thermophoresis, the effect of a plane boundary on the surrounding gas atom distribution in which there exists a constant temperature gradient at infinity is described by the well-known temperature jump problem $(10,11)$. In order to assess the effect of the boundary interaction, without going into detailed calculations, we shall consider the case where the gas can be represented by atoms which have a constant scattering cross section rather than constant collision frequency as would be the case for Maxwell molecules. The reason for this assumption is that an exact solution is available for the temperature profile in this case which can easily be incorporated into the expression for the force. In addition, we assume that the gas-surface interaction is governed solely by diffuse emission. These limitations can be relaxed at the expense of additional analytical and computational labor but at this stage we are concerned with general trends rather than specific details.

\section{THE FORCE ON A PARTICLE IN A GAS}

Force is the rate of change of momentum. Thus in order to calculate the net force on a particle in a nonuniform gas, we compute

$$
\mathbf{F}=-\left(\frac{\partial \mathbf{M}}{\partial t}\right)_{\text {coll }}
$$

where $\mathbf{M}$ is the momentum and the subscript "coll" denotes collision due to molecular impacts.

If $(\partial f / \partial t)_{\text {coll }}$ is the rate of change of the gas atom distribution function $f(\mathbf{v}, \mathbf{r})$ due to collisions, then by definition

$$
\mathbf{F}=-m \int d \mathbf{v}(\mathbf{v}-\mathbf{v})\left(\frac{\partial f}{\partial t}\right)_{\text {coll }},
$$

where $\mathbf{V}$ is the macroscopic velocity of the particle and $m$ is the mass of a molecule. In an earlier paper (12), the author has shown that

$$
\begin{aligned}
\left(\frac{\partial f}{\partial t}\right)_{\text {coll }}=\int d \mathbf{v}^{\prime} \sigma\left(\mathbf{v}^{\prime}-\mathbf{V}\right. & \rightarrow \mathbf{v}-\mathbf{V}) f\left(\mathbf{v}^{\prime}, \mathbf{r}\right) \\
& -|\mathbf{v}-\mathbf{V}| \sigma f(\mathbf{v}, \mathbf{r})
\end{aligned}
$$

where $\sigma\left(\mathbf{v}^{\prime} \rightarrow \mathbf{v}\right)$ is the global scattering kernel of the particle. $\sigma\left(\mathbf{v}^{\prime} \rightarrow \mathbf{v}\right)$ will depend upon the temperature of the particle and the nature of the gas-surface interaction, e.g. diffuse, specular, Lambert, backward scattering.

Inserting Eq. [3] into Eq. [2] and changing variables lead to

$$
\begin{aligned}
\mathbf{F}=m \sigma \int d \operatorname{gg} g f(\mathbf{g}+\mathbf{V}, \mathbf{r})-m \int d \mathbf{g g} \\
\times \int d \mathbf{g}^{\prime} \sigma\left(\mathbf{g}^{\prime} \rightarrow \mathbf{g}\right) f\left(\mathbf{g}^{\prime}+\mathbf{V}, \mathbf{r}\right) .
\end{aligned}
$$

If we write the scattering kernel as

$$
\sigma\left(\mathbf{g}^{\prime} \rightarrow \mathbf{g}\right)=\sum_{l=0}^{\infty} \frac{2 l+1}{4 \pi} \sigma_{l}\left(g^{\prime} \rightarrow g\right) P_{l}\left(\mathbf{\Omega}^{\prime} \cdot \mathbf{\Omega}\right)
$$

where $\mathbf{g}=g \boldsymbol{\Omega}$ and $\mathbf{g}^{\prime}=g^{\prime} \boldsymbol{\Omega}$ and let $\mathbf{n}$ be the unit vector in the direction of particle motion, we find that the force becomes

$$
\begin{array}{r}
\mathbf{F}=m \sigma \int_{0}^{\infty} d g g^{4} \int d \Omega \Omega f(g, \mathbf{n} \cdot \mathbf{\Omega}, \mathbf{r}) \\
-m \int_{0}^{\infty} d g g^{3} \int_{0}^{\infty} d g^{\prime} g^{\prime 2} \sigma_{1}\left(g^{\prime} \rightarrow g\right) \\
\times \int d \Omega^{\prime} \mathbf{\Omega}^{\prime} f\left(g^{\prime}, \mathbf{n} \cdot \mathbf{\Omega}^{\prime}, \mathbf{r}\right) .
\end{array}
$$


Now let us assume that we can write the dis- and tribution function $f$ as

$$
\begin{aligned}
& f(g, \mathbf{n} \cdot \mathbf{\Omega}, \mathbf{r})= n(z)\left[\frac{m}{2 \pi k T(x)}\right]^{3 / 2} \\
& \times \exp \left[-\frac{m}{2 k T(x)}(\mathbf{g}+\mathbf{V})^{2}\right] \\
& \\
& \times[1+h(g, \mathbf{n} \cdot \mathbf{\Omega}, x)] .
\end{aligned}
$$

In the temperature jump problem, we assume that asymptotically there exists a linear temperature gradient in the gas,

$$
T(x)=T_{0}(1+K x)
$$

and

$$
n(x)=\frac{n_{0}}{1+K x}
$$

such that $p=n k T$ is constant throughout and $K=(1 / T)(d T / d x)$.

For small gradients we can linearize the Maxwellian to obtain

$$
\begin{array}{r}
f(g, \mathbf{n} \cdot \Omega, \mathbf{r})=f_{0}(g)\left\{1-2 g V \mathbf{n} \cdot \Omega\left(\frac{m}{2 k T_{0}}\right)\right. \\
\left.+K x\left(\frac{m v^{2}}{2 k T_{0}}-\frac{5}{2}\right)+h(g, \mathbf{n} \cdot \Omega, x)\right\},
\end{array}
$$

where

$$
f_{0}(g)=n_{0}\left(\frac{m}{2 \pi k T_{0}}\right)^{3 / 2} \exp \left(-\frac{m g^{2}}{2 k T_{0}}\right)
$$

and $T_{0}$ is the temperature of the wall.

Inserting Eq. [8] into Eq. [6] leads to

$$
F_{\mathrm{n}}=F_{\mathrm{T}}+F_{\mathrm{V}} \text {, }
$$

where

$$
\begin{aligned}
& F_{\mathrm{V}}=-\frac{m}{2 k T_{0}} \cdot 2 m V\left\{\sigma \int_{0}^{\infty} d g g^{5} f_{0}(g)\right. \\
&\left.-\int_{0}^{\infty} d g g^{3} \int_{0}^{\infty} d g^{\prime} g^{\prime 3} \sigma_{1}\left(g^{\prime} \rightarrow g\right) f_{0}\left(g^{\prime}\right)\right\} \\
& \quad \times \int d \Omega(\mathbf{n} \cdot \mathbf{\Omega})^{2}
\end{aligned}
$$

$$
\begin{gathered}
F_{\mathrm{T}}=m \sigma \int_{0}^{\infty} d g g^{4} f_{0}(g) \int d \Omega(\mathbf{n} \cdot \mathbf{\Omega}) h(g, \mathbf{n} \cdot \mathbf{\Omega}, x) \\
-m \int_{0}^{\infty} d g g^{3} \int_{0}^{\infty} d g^{\prime} g^{\prime 2} \sigma_{1}\left(g^{\prime} \rightarrow g\right) f_{0}\left(g^{\prime}\right) \\
\quad \times \int d \Omega^{\prime}\left(\mathbf{n} \cdot \mathbf{\Omega}^{\prime}\right) h\left(g^{\prime}, \mathbf{n} \cdot \mathbf{\Omega}^{\prime}, x\right) .
\end{gathered}
$$

Physically, $F_{\mathrm{V}}$ is the frictional drag force on the particle as it moves with velocity $V$ in the gas and is the quantity discussed by Waldmann and Schmitt (5) and from a different viewpoint by Williams (6). $F_{\mathrm{T}}$, on the other hand, is due to the nonuniform velocity distribution in the gas caused by the temperature gradient (or by any other mechanism we choose). In the next section we shall demonstrate how to calculate $h$.

\section{THE VELOCITY DISTRIBUTION OF THE GAS MOLECULES IN A TEMPERATURE GRADIENT}

\subsection{The Transport Equation}

The Boltzmann equation for gases when linearized about the Maxwellian of Eq. [7] with $V=0$, leads to $(11,13)$

$$
\begin{aligned}
K c_{x}\left(c^{2}-\frac{5}{2}\right)+ & c_{\mathrm{x}} \frac{\partial}{\partial x} h(\mathbf{c}, x)+V(c) h(\mathbf{c}, x) \\
& =\int d \mathbf{c}^{\prime} K\left(\mathbf{c}, \mathbf{c}^{\prime}\right) e^{-c^{\prime 2}} h\left(\mathbf{c}^{\prime}, x\right),
\end{aligned}
$$

where $c^{2}=m v^{2} / 2 k T_{0}, V(c)$ is the collision frequency, and $K\left(\mathbf{c}, \mathbf{c}^{\prime}\right)$ the scattering kernel for gas atoms. If we write

$$
h(\mathbf{c}, x)=h(c, \mu, \chi, x) \text {, }
$$

where $\mu$ and $\chi$ are the polar angles describing the vector $\mathbf{c}$, then the boundary condition for Eq. [12] is written (13)

$$
\begin{aligned}
h(c, \mu, \chi, 0)= & \frac{2}{\pi} \int_{0}^{\prime} d \mu^{\prime} \mu^{\prime} \int_{0}^{2 \pi} d \chi^{\prime} \int_{0}^{\infty} \\
& \times d c^{\prime} c^{\prime 3} e^{-c^{\prime 2}} h\left(c^{\prime},-\mu^{\prime}, \chi^{\prime}, 0\right) \\
& \mu>0, \text { all } \chi .
\end{aligned}
$$


This condition assumes that molecules are reflected from the plane surface diffusely in a Maxwellian distribution characterized by the temperature of the surface.

In order to proceed it is necessary to introduce a model for gas-atom scattering. Kernels based upon physically realistic force laws do not lead to analytically tractable results, and it is common practice to use synthetic models which preserve the main physical characteristics of the scattering process and yet lead to analytical progress. Such a model is the velocity-dependent BGK model $(10,11)$ which conserves number, momentum, and energy and also preserves the correct velocity dependent of the collision frequency $V(c)$. In view of this we write

$$
K\left(\mathbf{c}, \mathbf{c}^{\prime}\right)=\sum_{l=0}^{\infty} \frac{2 l+1}{4 \pi} K_{l}\left(c, c^{\prime}\right) P_{l}\left(\hat{\mathbf{c}} \cdot \hat{\mathbf{c}}^{\prime}\right)
$$

with

$$
\begin{aligned}
& K_{0}\left(c, c^{\prime}\right)=\left\{\gamma_{0}+\gamma_{2}\left(c^{2}-\omega\right)\left(c^{2}-\omega\right)\right\} \\
& \times V(c) V\left(c^{\prime}\right) \\
& K_{1}\left(c, c^{\prime}\right)=\gamma_{1} c c^{\prime} V(c) V\left(c^{\prime}\right) \\
& K_{l}\left(c, c^{\prime}\right)=0 ; \quad l>1,
\end{aligned}
$$

where $\gamma_{0}^{-1}=V_{0}, \omega=V_{2} / V_{0}, \gamma_{1}^{-1}=V_{2}$, and $\gamma_{2}=V_{0} /\left(V_{0} V_{4}-V_{2}^{2}\right)$ with

$$
V_{n}=\int_{0}^{\infty} d c c^{n+2} e^{-c^{2}} V(c) .
$$

Inserting Eqs. [15]-[18] into Eq. [12] and assuming that $V(c)=c \Sigma$, where $\Sigma$ is independent of $c$, and, after integrating over $\chi(0,2 \pi)$, we get the following linear transport equation

$$
\begin{aligned}
& K\left(c^{2}-\frac{5}{2}\right) \mu+\left(\mu \frac{\partial}{\partial x}+\Sigma\right) p(c, \mu, x) \\
& =\frac{1}{2} \Sigma \int_{0}^{\infty} d c^{\prime} c^{\prime 3} e^{-c^{\prime 2}}\left\{2+\left(c^{2}-2\right)\left(c^{\prime 2}-2\right)\right\} \\
& \quad \times \int_{-1}^{1} d \mu^{\prime} p\left(c^{\prime}, \mu^{\prime}, x\right)+\frac{3}{2} \mu c \Sigma \int_{0}^{\infty} d c^{\prime} c^{\prime 4} e^{-c^{\prime 2}} \\
& \quad \times \int_{-1}^{1} d \mu^{\prime} \mu^{\prime} p\left(c^{\prime}, \mu^{\prime}, x\right),
\end{aligned}
$$

where

$$
p(c, \mu, x)=\frac{1}{2 \pi} \int_{0}^{2 \pi} d \chi h(c, \mu, \chi, x) .
$$

The boundary condition [14] becomes

$$
\begin{aligned}
p(c, \mu, 0)=4 \int_{0}^{1} d \mu^{\prime} \mu^{\prime} \int_{0}^{\infty} d c^{\prime} c^{\prime 3} e^{-c^{\prime 2}} & \\
& \times p\left(c^{\prime},-\mu^{\prime}, 0\right) \quad \mu>0 .
\end{aligned}
$$

In terms of $p(c, \mu, x)$, the force on the particle can be written

$$
\begin{aligned}
& F_{\mathrm{Tx}}=\frac{2 m n_{0}}{\sqrt{\pi}} \frac{2 k T_{0}}{m}\left\{\sigma \int_{0}^{\infty} d c c^{4} e^{-c^{2}}\right. \\
& \times \int_{-1}^{1} d \mu \mu p(c, \mu, x)-\int_{0}^{\infty} d c c^{3} \int_{0}^{\infty} d c^{\prime} c^{\prime 2} e^{-c^{\prime 2}} \\
& \left.\quad \times \tilde{\sigma}_{1}\left(c^{\prime} \rightarrow c\right) \int_{-1}^{1} d \mu^{\prime} \mu^{\prime} p\left(c^{\prime}, \mu^{\prime}, x\right)\right\},
\end{aligned}
$$

where

$$
\sigma_{1}\left(g^{\prime} \rightarrow g\right)=\frac{m}{2 k T_{0}} \tilde{\sigma}_{1}\left(c^{\prime} \rightarrow c\right) .
$$

\subsection{The Solution by Wiener-Hopf Technique}

Equation [19] has been solved for certain angular moments of interest in the temperature jump problem in a previous paper (14). Thus it is not necessary to repeat all of the analysis here. Suffice to say we seek a solution to Eq. [19] in the form

$$
\begin{aligned}
p(c, \mu, x)=p_{0}(\mu, x) & \\
& +c p_{1}(\mu, x)+c^{2} p_{2}(\mu, x)
\end{aligned}
$$

when the force in Eq. [22] becomes

$$
\begin{aligned}
F_{\mathrm{TX}}=\frac{4 k n_{0} \sigma T_{0}}{\sqrt{\pi}} & \left\{\partial_{0} p_{01}(x)\right. \\
& \left.+\delta_{1} p_{11}(x)+\phi_{2} p_{21}(x)\right\},
\end{aligned}
$$

where

$$
\begin{aligned}
\delta_{0}=\frac{3 \sqrt{\pi}}{8}-\frac{1}{\sigma} \int_{0}^{\infty} & d c c^{3} \int_{0}^{\infty} \\
& \times d c^{\prime} c^{\prime 2} e^{-c^{2} \tilde{\sigma}_{1}\left(c^{\prime} \rightarrow c\right)}
\end{aligned}
$$




$$
\begin{aligned}
& \nexists_{1}=1-\frac{1}{\sigma} \int_{0}^{\infty} d c c^{3} \int_{0}^{\infty} \\
& \quad \times d c^{\prime} c^{\prime 3} e^{-c^{\prime 2}} \tilde{\sigma}_{1}\left(c^{\prime} \rightarrow c\right) \\
& g_{2}=\frac{15 \sqrt{\pi}}{16}-\frac{1}{\sigma} \int_{0}^{\infty} d c c^{3} \int_{0}^{\infty} \\
& \times d c^{\prime} c^{\prime 4} e^{-c^{\prime 2}} \tilde{\sigma}_{1}\left(c^{\prime} \rightarrow c\right) .
\end{aligned}
$$

The problem reduces therefore to the calculation of the angular moments $p_{01}, p_{11}$, and $p_{21}$. From our previous work, we know that

$$
\begin{gathered}
p_{21}(x)+\frac{3 \sqrt{\pi}}{16} p_{11}(x)=-\frac{3 K}{4 \Sigma} \\
p_{01}(x)-2 p_{21}(x)=\frac{3 K}{\Sigma} .
\end{gathered}
$$

Hence we can write

$$
F_{\mathrm{Tx}}=\frac{4 n_{0} \sigma k T_{0}}{\sqrt{\pi}} \mathcal{F}(x)
$$

where

$$
\begin{aligned}
\mathcal{F}(x)= & \frac{3 K}{4 \Sigma}\left(2 \partial_{0}-\partial_{2}\right) \\
& +\left(\partial_{1}-\frac{3 \sqrt{\pi}}{8} \partial_{0}-\frac{3 \sqrt{\pi}}{16} \partial_{2}\right) p_{11}(x) .
\end{aligned}
$$

At this point it is interesting to note that for all of the models of gas-particle interaction (6), e.g.,

Specular $\quad \tilde{\sigma}_{1}\left(c^{\prime} \rightarrow c\right)=0$

$$
\text { Diffuse } \quad \tilde{\sigma}_{1}\left(c^{\prime} \rightarrow c\right)=-\frac{8}{9} \sigma c c^{\prime} e^{-c^{2}}
$$

Lambert $\quad \tilde{\sigma}_{1}\left(c^{\prime} \rightarrow c\right)=-\frac{4 \sigma}{9 c} \delta\left(c-c^{\prime}\right)$

Backward $\quad \tilde{\sigma}_{1}\left(c^{\prime} \rightarrow c\right)=-\frac{\sigma}{c} \delta\left(c-c^{\prime}\right)$,

the function $\mathcal{F}(x)$ can be written

$$
\mathcal{F}(x)=Q\left[-\frac{9 \sqrt{\pi} K}{64 \Sigma}+\frac{\lambda}{3} p_{11}(x)\right],
$$

where $\lambda=3(1-81 \pi / 256)$ and $Q$ takes the following values:

\section{Specular 1 \\ Diffuse 1 \\ Lambert $13 / 9$ \\ Backward 2.}

The equation for $p_{1}(\mu, x)$ is found from our previous work to be

$$
\begin{aligned}
{\left[\mu \frac{\partial}{\partial x}+\Sigma\right] p_{1}(\mu, x) } & \\
= & \frac{\lambda}{2} \Sigma \mu p_{11}(x)-\frac{27 \sqrt{\pi}}{128} \mu K
\end{aligned}
$$

subject to the boundary condition

$$
p_{1}(\mu, 0)=0 ; \quad \mu>0 \text {. }
$$

The solution of this equation is obtained using standard Wiener-Hopf techniques (11). The only additional information required is the value of $p_{11}(\infty)$ which can be obtained by noting that at very large distances from the plane surface, the force on the particle will be the isolated sphere value already obtained by Waldmann and Schmitt (5). Thus we write the distribution function as

$p(c, \mu, x)=B_{0}\left(c^{2}-\frac{5}{2}\right)-K c a(c) \mu$

$$
-\rho(c, \mu, x) \text {, }
$$

where $\rho$ is a boundary transient which rapidly becomes zero as we move away from the surface. $a(c)$ is a solution of the classical Chapman-Enskog integral equation (13) which for the case of a constant cross section BGK model leads to

$$
c a(c)=\frac{2 c}{3 \sqrt{\pi} \Sigma}+\frac{1}{\Sigma}\left(c^{2}-\frac{5}{2}\right)
$$

Hence

$$
\begin{aligned}
\int_{-1}^{1} d \mu \mu p(c, \mu, \infty) & =p_{01}(\infty)+c p_{11}(\infty) \\
+c^{2} p_{21}(\infty) & =\frac{2 K}{3 \Sigma}\left\{\frac{5}{2}-\frac{2 c}{3 \sqrt{\pi}}-c^{2}\right\}
\end{aligned}
$$

and therefore

$$
p_{11}(\infty)=-\frac{4 K}{9 \sqrt{\pi} \Sigma}
$$


With this result we can write for $p_{11}(x)$

$$
\begin{aligned}
& p_{11}(x)=-\frac{4 K}{9 \sqrt{\pi} \Sigma} \\
&+\frac{3 K}{4 \lambda \Sigma} e^{-\alpha x} \frac{\left(1-\alpha^{2}\right)}{H_{1}(1 / \alpha)\left[\lambda-3\left(1-\alpha^{2}\right)\right]}+\frac{3 K}{8 \Sigma} \\
& \quad \times \int_{0}^{1} d \mu \mu^{2} e^{-x \Sigma / \mu} \frac{1}{H_{1}(\mu) Z_{1}(\mu)} .
\end{aligned}
$$

We may also show that the exact value of $p_{11}(0)$ is

$$
p_{11}(0)=-\frac{3 K}{4 \lambda \Sigma}\left(1-\frac{9 \sqrt{\pi}}{16}\right) .
$$

The quantities in Eq. [41] are

(a) $\alpha$ is the real root of

$$
1+\frac{\lambda}{\alpha^{2}}-\frac{\lambda}{2 \alpha^{3}} \log \left(\frac{1+\alpha}{1-\alpha}\right)=0
$$

(b) $H_{1}(\mu)$ is the Chandrasekhar $H$-function based on the equation $(11,15)$

$$
M_{1}(s)=1+\frac{\lambda}{s^{2}}-\frac{\lambda}{2 s^{3}} \log \left(\frac{1+s}{1-s}\right)
$$

(c)

$$
\begin{array}{r}
Z_{1}(\mu)=\left[1+\lambda \mu^{2}+\frac{\lambda}{2} \mu^{3} \log \left(\frac{1+\mu}{1-\mu}\right)\right]^{2} \\
+\frac{\pi^{2}}{4} \lambda^{2} \mu^{6}
\end{array}
$$

It is interesting to note that the root $\alpha=1$ $+O\left(e^{-40}\right)$, thus for a very good approximation indeed we may neglect the exponential term in Eq. [41]. Moreover, if we set $H_{1}$ and $Z_{1}$, in the integrand of Eq. [41], equal to unity, we can write

$$
p_{11}(x)=-\frac{4 K}{9 \sqrt{\pi} \Sigma}+\frac{3 K}{8 \Sigma} E_{4}(\Sigma x)
$$

$E_{4}(x)$ being an exponential integral. The accuracy of this approximation can be checked at its worst point, namely at $x=0$, by comparison with the exact value given by Eq. [42]. We find

$$
p_{11}(0)=-\frac{K}{\Sigma}\left(\frac{4}{9 \sqrt{\pi}}-\frac{1}{8}\right)=-0.12575 \frac{K}{\Sigma}
$$

for the approximate value, and for the exact one,

$$
p_{11}(0)=-0.125186 K / \Sigma \text {. }
$$

Thus the error is very small and we shall use Eq. [46]. Inserting $p_{11}(x)$ into the expression for the force leads to

$$
\mathcal{F}(x)=Q \frac{K}{\Sigma}\left[-\frac{4}{9 \sqrt{\pi}}+\frac{3}{8}\left(1-\frac{81 \pi}{256}\right) E_{4}(\Sigma x)\right] \text {. }
$$

We can obtain, therefore, the values of $\mathcal{F}(x)$ at the surface and at infinity (using the exact value of $\left.p_{11}(0)\right)$, viz.,

$$
\begin{gathered}
\mathcal{F}(0)=-\frac{Q K}{4 \Sigma} \\
\mathcal{F}(\infty)=-\frac{4 Q K}{9 \sqrt{\pi}} .
\end{gathered}
$$

Hence

$$
\frac{\mathscr{F}(0)}{\mathcal{F}(\infty)}=\frac{9 \sqrt{\pi}}{16}=0.9970052 \cdots
$$

We may conclude therefore that the effect of the surface is very small and that the effect is negligible at a distance of about three mean free paths from the surface.

Finally we write the thermophoretic force in the form

$$
\begin{aligned}
F_{\mathrm{T} x}=-\frac{32}{27 \sqrt{\pi}} & Q \sigma \lambda_{\mathrm{T}}\left(\frac{m}{2 k T_{0}}\right)^{1 / 2} \\
& \times \nabla T\left[1-\frac{9 \lambda \sqrt{\pi}}{32} E_{4}(\Sigma x)\right],
\end{aligned}
$$

where we have used the following relationship between the mean free path $1 / \Sigma$ and the thermal conductivity $\lambda_{\mathrm{T}}$, viz.,

$$
\frac{1}{\Sigma}=\frac{2 \sqrt{\pi}}{3} \frac{\lambda_{\mathrm{T}}}{n_{0} k}\left(\frac{m}{2 k T_{0}}\right)^{1 / 2} \text {. }
$$

If we evaluate the drag force in Eq. [10] and set $F_{\mathrm{T}}+F_{\mathrm{V}}=0$ we can obtain the thermophoretic velocity as 


$$
V=-\alpha \frac{2}{9} \frac{\lambda_{\mathrm{T}}}{p} \nabla T\left[1-\frac{9 \sqrt{\pi} \lambda}{32} E_{4}(\Sigma x)\right],
$$

where for all models $\alpha=1$ except for diffuse scattering between sphere and gas when $\alpha$ $=1 /(1+\pi / 8)$.

\section{CONCLUDING REMARKS}

The effect of a plane surface on the thermophoretic force on an approaching particle in the Knudsen regime is seen to be very small. Although the calculations in this paper have been carried out for a constant collision cross section rather than for the more appropriate constant collision frequency, it is unlikely that this will dramatically affect the outcome, although the extent of its influence may be somewhat larger. Similarly, the assumption of diffuse reflection at the plane surface is likely to lead to the most severe distortions of the velocity distribution function and hence to the largest influence on the force. In spite of the smallness of the effect on the thermophoretic force, it is in fact finite, and it is instructive to explain it physically. The formula [51] shows that, in the neighborhood of the plane surface, the thermophoretic force is decreased. Since the phenomenon of thermophoresis is due to the difference between the rates of molecular impact on the hot and cold sides of the particle, we may conclude that in the neighborhood of the wall there is an additional contribution arising from the boundary condition [14]. In fact molecules are coming directly from the wall and striking the particle before being thermalized in the bulk gas. Thus on the cold side the impacts are dominated by the characteristics of the wall rather than the properties of the bulk gas through the function $a(c)$. However, these direct transport effects are only dominant over a region of about one or two mean free paths from the wall and this is borne out by the presence of the rapidly attenuating function $E_{4}(\Sigma x)$.

\section{REFERENCES}

1. Friedlander, S. K., "Smoke, Dust \& Haze.” Wiley, New York, 1977.

2. Derjaguin, B. V., and Yalamov, Yu. I., in "Topics in Current Aerosol Research" (M. Hidy and J. R. Brock, Eds.), Vol. 3, Part 2. Pergamon, Elmsford, NY, 1972.

3. Dwyer, H. A., Phys. Fluids 10, 976 (1967).

4. Phillips, W. F., Phys. Fluids 18, 144 (1975).

5. Waldmann, L., and Schmitt, K. H., in "Aerosol Science" (C. N. Davies, Ed.). Academic Press, New York, 1966.

6. Williams, M. M. R., Z. Naturforsch. 27, 1798 (1972).

7. Epstein, P. S., Z. Phys. 54, 537 (1929).

8. Brock, J. R., J. Colloid Sci. 17, 768 (1962).

9. Williams, M. M. R., submitted.

10. Cercignani, C., "Theory and Application of the Boltzmann Equation." Academic Press, Edinburgh, 1975.

11. Williams, M. M. R., "Mathematical Methods in Particle Transport Theory," Butterworths, London, 1971.

12. Williams, M. M. R., Z. Naturforsch. 27, 1804 (1972).

13. Williams, M. M. R., J. Fluid Mech. 45, 759 (1971).

14. Cassell, J. S., and Williams, M. M. R., Transp. Theory Stat. Phys. 2(1), 81 (1972).

15. Chandrasekhar, S., "Radiative Transfer." Dover, New York, 1960. 